\title{
Frontal Dysexecutive Syndrome in Brain Tumors: A Pragmatic Insight to an Old Problem
}

\author{
Souvik Dubey ${ }^{a} \quad$ Ritwik Ghosh $^{\mathrm{b}} \quad$ Subhankar Chatterjee ${ }^{c}$ \\ Mahua Jana Dubey ${ }^{d}$ Samya Sengupta ${ }^{e}$ Subham Chatterjee ${ }^{f}$ \\ Biman Kanti Ray ${ }^{a}$ Pedro J. Modrego ${ }^{g}$ Julián Benito-León ${ }^{\text {h, } i, j}$ \\ aDepartment of Neuromedicine, Bangur Institute of Neurosciences, Kolkata, India; \\ bDepartment of General Medicine, Burdwan Medical College and Hospital, Burdwan, India; \\ 'Department of General Medicine, Rajendra Institute of Medical Sciences, Ranchi, India; \\ ${ }^{\mathrm{d} D e p a r t m e n t}$ of Psychiatry, Berhampore Mental Hospital, Murshidabad, India; 'Department \\ of General Medicine, Apollo Gleneagles Hospitals, Kolkata, India; ' Department of Psychiatry, \\ Institute of Psychiatry, Institute of Post-Graduate Medical Education and Research, Kolkata, \\ India; 9Department of Neurology, University Hospital Miguel Servet, Zaragoza, Spain; \\ hDepartment of Neurology, University Hospital "12 de Octubre", Madrid, Spain; 'Centro \\ de Investigación Biomédica en Red sobre Enfermedades Neurodegenerativas (CIBERNED), \\ Madrid, Spain; jDepartment of Medicine, Complutense University, Madrid, Spain
}

\section{Keywords}

Brain tumors - Space-occupying lesion - Frontal dysexecutive syndrome - Diaschisis .

Plasticity $\cdot$ Cognitive impairment

\begin{abstract}
Brain tumors have long been considered one of the most prevalent causes of potentially reversible cognitive impairment. An accurate underlying cause of cognitive impairment due to brain tumor needs to be evaluated pragmatically. Patterns of cognitive impairment associated with brain tumors depend mainly on their location, lateralization, pathological classification and secondary effects of the treatment, as well as the structural plasticity and diaschisis. Hence, it is not rare that lesions with different locations and histologies may manifest with a similar pattern of cognitive impairment due to the complex interplay of determinants. We herein report 3 patients with brain tumors affecting different locations and with differing histologies, who shared a similar presentation as "frontal dysexecutive syndrome" masqueraded as psychiatric conditions. Detailed examination of saccades and pursuit along with eye movements and conventional motor examinations were essential not only to diagnose brain tumor as the potential cause of cognitive impairment, but also to rule out other coexisting etiologies
\end{abstract}


with completely different underlying pathological mechanisms (i.e., Huntington's disease in 1 of the cases). A detailed neurological examination, including eye movement assessment, in patients with psychiatric symptoms provides not only important clues to delineate the underlying anatomical substrate involved, but also helps clinicians to make an accurate diagnosis and to select appropriate therapeutic options.

\section{Introduction}

Brain tumors have long been considered one of the most prevalent causes of potentially reversible cognitive impairment [1]. An accurate underlying cause of cognitive impairment due to brain tumors needs to be evaluated pragmatically [2]. Patterns of cognitive impairment associated with brain tumors depend mainly on their location, lateralization, pathological classification and secondary effects of the treatment, as well as the structural plasticity and diaschisis [3]. Hence, it is not rare that lesions with different locations and histologies may manifest with similar patterns of cognitive impairment due to the complex interplay of determinants [2].

We herein report 3 patients with brain tumors in different locations and with varying characteristics, who shared a similar presentation as "frontal dysexecutive syndrome" masqueraded as psychiatric disorders. Detailed examination of saccades and pursuit along with eye movements and conventional motor examination were essential not only to diagnose brain tumor as the potential causes of cognitive impairment, but also to rule out other coexisting etiologies with completely different underlying pathological mechanisms (i.e., Huntington's disease) in 1 of the cases.

\section{Cases Presentation}

\section{Patient 1}

A 27-year-old woman presented with the complaint of progressively making mistakes in her daily activities for the last 2 years. Her husband revealed that he had noticed his wife becoming less attentive to his words over this period. She often forgot the topic of conversation midway through. Day-by-day, it became difficult for her to keep track during telephone conversations. Recently, dishes prepared by her were becoming inedible as she forgot to add important ingredients. Her behavior at this time was marked by unusual restlessness, as noticed by a caregiver. Her attitude/behavior seemed vacant in situations demanding planning or decision making in day-to-day activities. She showed distress when having to initiate any work requiring some motivation. Infrequently, she repeated the same phrases/ words, and sometimes continued doing same thing repeatedly. However, to date there was no history suggestive of any disinhibition or abnormal sexual behavior. There was history of forgetfulness of recent events in the form of frequent misplacement of objects. It was also difficult for her to do shopping. She even sought for food after having just served a meal. She mixed up days, dates, and looked easily confused even by trivial day to day commands. There was history of difficulties in operating gas ovens, and forgetfulness to switch off lights or fans when not in use. Her comprehension of spoken vernacular language was normal. There was no deficit in the expression of language, fluency, grammar, and repetition. No paraphasia or neologism were noted during conversation. No history was suggestive of any difficulties in handling tools (no apraxia), any recognition problem (object or face), or difficulties in identi-

\section{Karger'}


fying a person by their name (no anomia). She could dress herself on her own. Marked distress was noted in her day-to-day money handling. Her bladder, bowel, sleep, and appetite remained normal. For the previous 5 months she had difficulty in hearing with her left ear and some abnormal sensation over the left side of her face, which was soon followed by a tendency to fall on the left side while walking. Her husband had also noticed some abnormal involuntary dancing movements involving the bilateral upper limb over the last year. On asking, it was revealed that her father had died at the age of 60 years and had also shown such movements since his fifties, with some behavioral abnormalities.

On examination, her mini mental status examination (MMSE) score was 16/30. Her attention (digit span and "A" vigil test) was impaired. Tests for executive function, such as trail making ("A" and "B"), alternating patterns, and motor Luria, were also impaired. Her frontal assessment battery (FAB) score was less than 12. As her attention was markedly impaired, word list recall (immediate and delayed) could not be done properly; however, during a word recall memory test, retrieval by cues was noted. Semantic and visual memories were preserved. Language testing was normal with tests for praxis and gnosis completed without impairment (however, during real object testing some impairments in the sequencing of given actions were noted). Simple reading did not show abnormalities (no dyslexia). Writing was impaired due to abnormal movements of the right upper limb (no grammatical mistakes or spelling errors). Preservative and repetitive behaviors were noted. There was no delusion, hallucination, hyperorality, physical or verbal disruptive behavior, simultanagnosia, or neglect.

A cranial nerve examination revealed increased saccadic latency, with hypometric and slow saccades. Anti-saccades were grossly impaired with broken pursuits and intermittent saccadic intrusions. There was also trigeminal sensory impairment over the V2 and V3 regions, while trigeminal motor functions, jaw jerk, and corneal reflexes were normal. Leftsided sensory-neural deafness was otherwise present. A cerebellar examination revealed left-sided intention tremor, meanwhile a left- sided heel shin test was impaired with generalized limb hypotonia. Power of all four limbs was normal with preserved deep tendon reflexes. Involuntary, flowing, dancing distal to proximal semi-purposeful movements were observed involving all 4 limbs (upper limbs more than lower limbs), suggestive of chorea. There was no sign of autonomic instability.

Brain magnetic resonance imaging (MRI) revealed a left cerebellopontine angle meningioma (Fig. 1) (later confirmed by histopathology), bilateral caudate atrophy, and mild dilatation of the anterior horns of lateral ventricles. The left cerebellopontine angle meningioma explained her trigeminal, vestibule-auditory, and left-sided cerebellar signs and symptoms. However, both the dilatation of anterior horns of the lateral ventricle and her frontal dysexecutive syndrome were not explained by the tumor. The genetics test for Huntington's disease was positive.

\section{Patient 2}

A right-handed 70-year-old man, with occasional alcohol abuse and 12 years of formal education, presented with his son as he was progressively becoming aloof from family concerns. His family members had noticed a change in his personality for last 1 year. Previously, he was highly active and well versed with familial concerns as well as with his business. For the last year he seemed to have withdrawn himself from his own family and liked spending most of the time alone, remaining quiet. He seemed to be apathetic in any familial or business concerns. He would only take foods on request. For last 6 months the patient had also significant progressive difficulties in walking, with a tendency to sway to the right side.

He looked inattentive throughout the whole conversation. He had marked distress in planning, decision making, organizing something, and sequencing of any activities. His under-

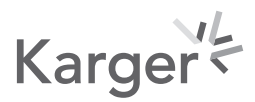




\section{in Oncology}

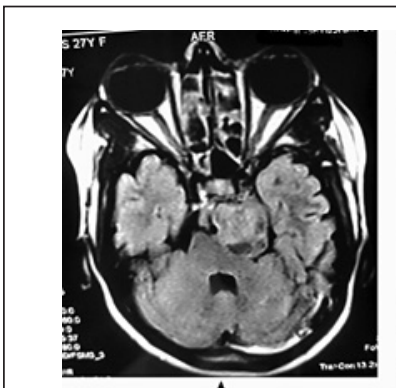

A

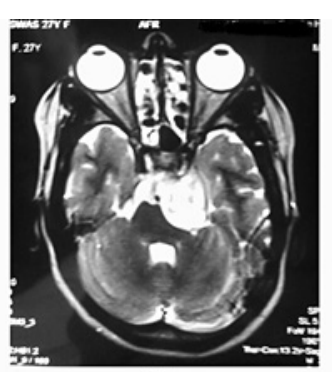

B

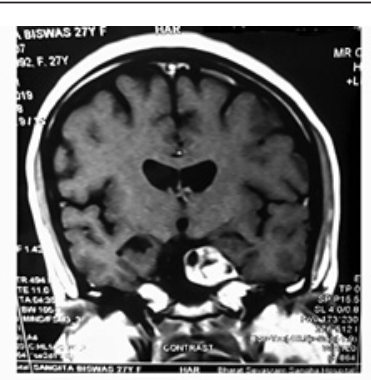

$\mathrm{C}$

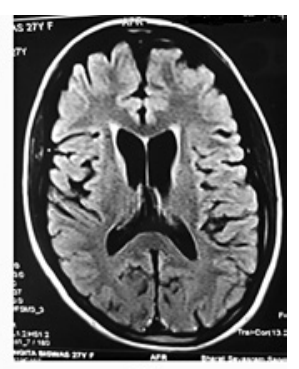

D

Fig. 1. Axial T1-weighted (A), axial-T2-weighted (B) and contrast-enhanced coronal T1-weighted (C) MRI images show a well-defined lobulated dumbbell-shaped extra-axial mass in the left prepontine cistern, measuring $2.27 \mathrm{~cm}(\mathrm{CC}) \times 2.83 \mathrm{~cm}(\mathrm{Tr}) \times 2.99 \mathrm{~cm}(\mathrm{AP})$ with multiple necrotic areas within. The lesion, which extends anteriorly to the left Meckel's cave and causes compression on the left side of the midbrain and pons, is suggestive of meningioma. Contrast-enhanced coronal T1-weighted (C) and axial T1-weighted (D) images also show bilateral caudate atrophy and mild dilatation of the anterior horns of lateral ventricles.

standing of spoken language revealed no abnormality, though verbal expressions seemed inadequate (without grammatical or syntax errors and no paraphasia or dysarthria). He had no difficulties in reading or writing. There was no visuospatial disorientation, or difficulties in naming or recognizing objects and faces. However, intermittently he forgot details of recent events, such as whether he had eaten, and was misplacing useful objects, but soon retrieved recollection on cues. No physical, disruptive, hallucinatory, or delusionary behavior, disinhibition or shameless bladder were noted. Cognitive screening revealed an MMSE score of 18/30. Detailed cognitive examination by vernacular adaptation of Addenbrooke's Cognitive Examination (ACE-III) was suggestive of attention deficit, executive dysfunction, and recent memory impairment. However, attention deficit significantly restrained comprehensive testing of the affected domains exclusively. His FAB score was less than 12 .

Cranial nerve examination revealed increased saccadic latency, hypometric saccades, and broken pursuit. Other cranial nerve examinations were non-contributory, including absence of nystagmus. The power of all 4 limbs was normal with hypertonia and brisk deep tendon reflexes. Right-sided cerebellar signs were obvious. Gait analysis revealed a broadbased cerebellar gait with a tendency to sway to the right side. His bilateral planter response was equivocal. Sensory and autonomic examinations were essentially normal. Brain MRI revealed a right-sided cerebellar hemangioblastoma with compression over the fourth ventricle resulting in secondary hydrocephalus (Fig. 2).

\section{Patient 3}

A right-handed 62-year-old woman, with 10 years of formal education, was admitted with cognitive impairment complaints for the last 3 years. Her daughter noticed that she had planning, organization, and sequencing difficulties, even with trivial matters, in day-to-day activities, more so in complex activities, which she used to do with ease before. She had difficulties in problem solving, indecisiveness, and seemed to be very rigid on any given situation or topic. She had progressive difficulties in keeping track of day-to-day conversation with easy distractibility. She started forgetting details of recent events and had a tendency to ask the same questions repeatedly. There was a history of frequent misplacement of objects. Furthermore, she repeatedly forgot to switch off the lights, fans, or gas oven when not in use. However, there was no history of difficulties in understanding of spoken language and her 


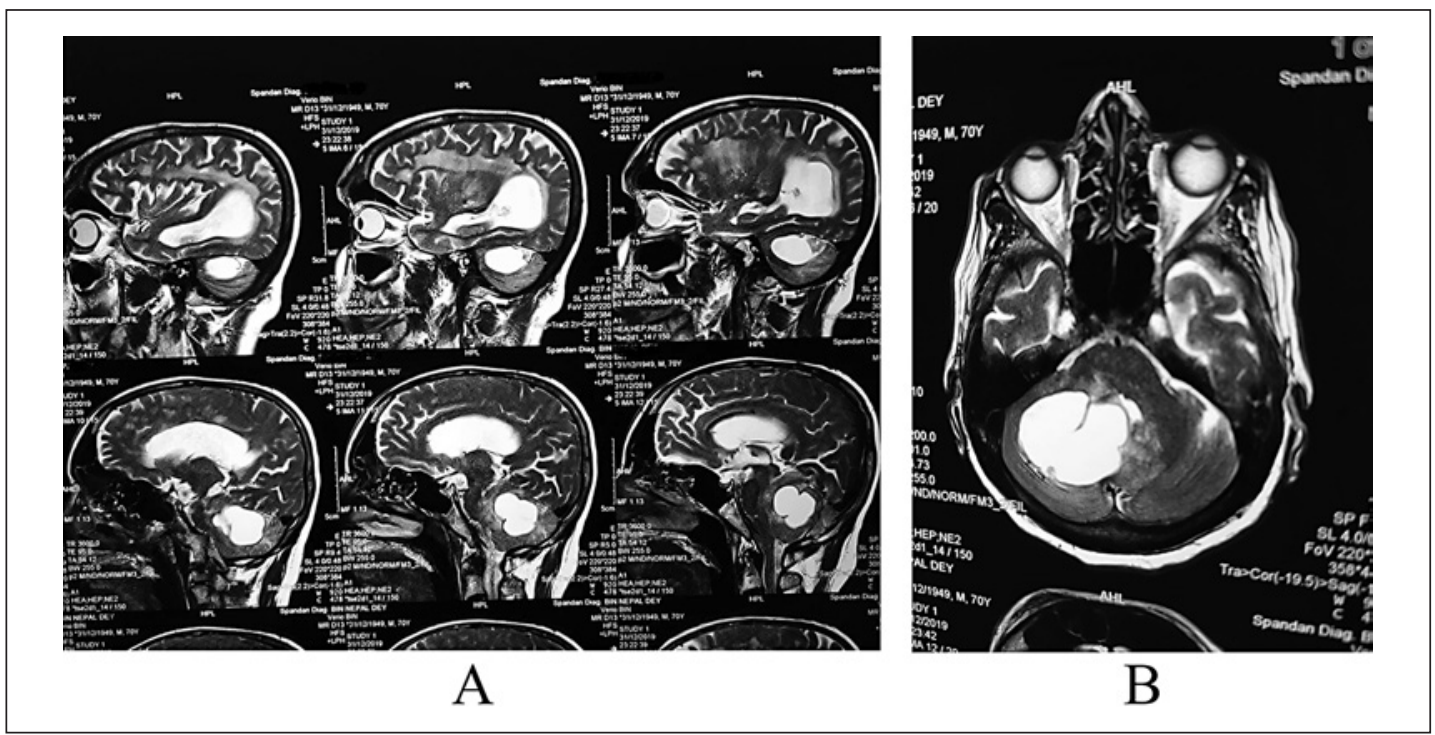

Fig. 2. Sagittal T2-weighted (A) and axial-T2-weighted (B) MRI images reveal a large, heterogenous lesion on the right side of the cerebellum with nodular hypointensity inside and pressure effects on the brainstem and cerebellum.

expressive verbal and non-verbal communications revealed no obvious abnormality. There was no history of difficulties in reading and writing, object or face recognition, naming, or visuospatial orientation. Hallucinations, delusions, disinhibition, hyperorality, hypersexuality, and physical and verbal disruptive behaviors were absent. Apart from her higher order cognitive deficits, she had walking difficulty and complained of occasional stiffness in her bilateral lower limbs. Her family history did not reveal any known neurological disease.

Examination revealed an MMSE score of 20/30. A detailed cognitive evaluation applying ACE-III revealed that the patient had attention deficit, executive dysfunction, and subcortical type memory impairment. Her FAB score was less than 12. Cranial nerve examination showed increased saccadic latency, hypometric slow saccades, broken pursuit, and vertical gaze restriction. The remaining cranial nerve examinations were normal. Speech and language examination were essentially normal. Motor examination revealed increased tone in the bilateral lower limbs. However, power in all 4 limbs were 5/5 (Medical Research Council grading). Deep tendon reflexes were normal in the upper limbs and brisk in the lower limbs. Planter responses were bilaterally equivocal. Her cerebellar and sensory examinations were all within normal limits with spastic gait. A brain MRI revealed a tectal plate glioma with hydrocephalus (Fig. 3).

\section{Discussion}

Our 3 patients with brain tumors presented with a frontal dysexecutive syndrome that mimicked different psychiatric disorders. In each case, there was a frontal subcortical network (cortico-striatal circuit) involvement with a similar clinical presentation to that seen in other pathologies, such as vascular dementia [4], Alzheimer's disease [4], Huntington's disease [5], normal pressure hydrocephalus [6], progressive supranuclear palsy [7], and HIV-associated neuro-degeneration [8], among others. 


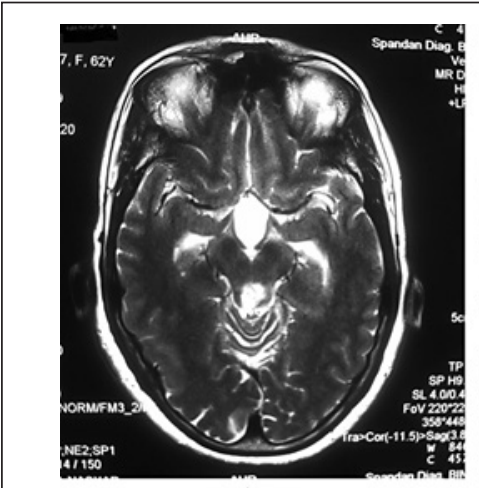

A

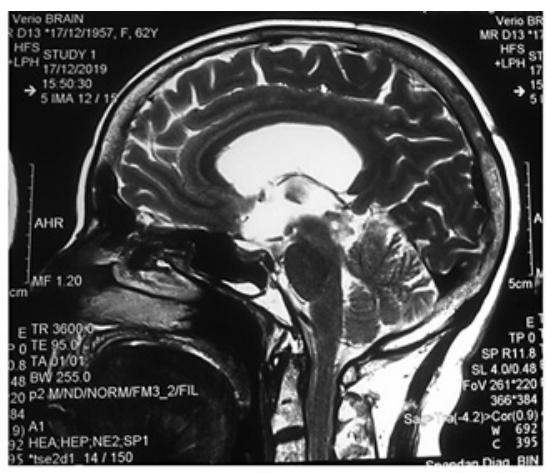

B

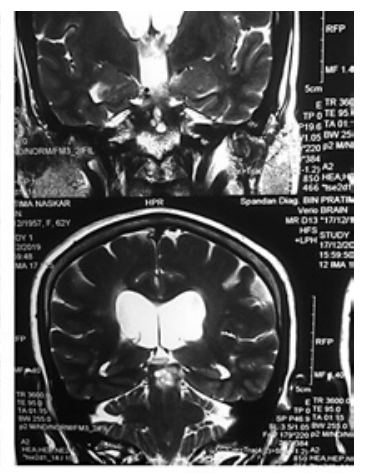

C

Fig. 3. Multi-planar axial T2-weighted (A), midsagittal (B), and coronal (C) MRI images reveal expansion of the tectal plate by a hyperintense, slow-growing, solid nodule of tissue compressing over the aqueduct and resulting in dilatation of supratentorial ventricles.

Our cases demonstrate that frontal subcortical network involvement may occur by different pathophysiological mechanisms and in varying anatomical locations. Firstly, it may occur due to obstructive hydrocephalus leading to disruption of cerebellocortical circuitry (tectal plate glioma) [9]. Secondly, it may be due to cerebellum damage, whereby patterns of cognitive impairment (executive dysfunction) and affect dysregulation may present as pseudo-frontal disorders, which confirms the functional association between these two central nervous system structures [10]. Thirdly, it may be involved by completely different pathophysiological processes (Huntington's disease and left cerebellopontine angle meningioma), which run in parallel ways [11].

The categorization of cognitive impairment is of utmost significance regarding outcome, reversibility, and use of therapeutic armamentarium in a pragmatic way. For example, cognitive impairment in the patient with Huntington's disease and meningioma is not going to improve after resection of the tumor. In contrast, shunt surgery may have some role in the improvement of cognitive status in the patient with tectal plate glioma [12-16].

The fact that should be emphasized in all 3 cases is that the clinical manifestations were initially subtle, mimicking known psychiatric disorders such as schizophrenia, depression, or bipolar affective disorder. Consequently, diagnosis was delayed in all 3 cases, which underscores the importance of searching for an underlying organic basis in any patient with psychiatric symptoms and dysexecutive syndrome [14-16]. The same holds true for subacute to acute onset psychiatric abnormalities like psychosis not otherwise specified, schizophrenia, schizophreniform illness, and mood disorders, which may masquerade as autoimmune disorders, deposition disorders, and thyroid-related disorders, among others $[15,17,18]$.

We highlight the performance of a detailed clinical examination of eye movements in patients with dysexecutive syndrome [19-22]. Our 3 patients had abnormalities of saccades, anti-saccades, and pursuit to vertical gaze. The anti-saccade task in the case of Huntington's disease, the broken pursuit in the case of the cerebellar hemangioblastoma, and restrictions of vertical gaze in the case of tectal plate glioma not only helped lesion localization, but also contributed to the recognition of the basic underlying pathophysiology of the lesions.

Motor involvement in "frontal dysexecutive syndrome" due to cortico-striatal involvement cannot be ignored. The involvement of bilateral periventricular white matter tracts (which include extra pyramidal and pyramidal tracts, and striatum and its connections) are in turn 
responsible for cognitive dysfunction as well as for motor manifestations (signs and symptoms of pyramidal tract involvement and movement disorders). Our cases are unique in the sense that they demonstrate the widespread nature of motor involvement, which ranges from movement disorders, such as chorea (due to concomitant extrapyramidal tract involvement) and hemiataxia (due to the left cerebellar hemisphere meningioma), to increased tone in the bilateral lower limbs because of brainstem and bilateral subcortical periventricular white matter tract involvement (due to obstructive hydrocephalus caused by tectal plate glioma).

Frontal dysexecutive syndrome not only warrants the examination of pyramidal, extrapyramidal, and cerebellar system. Besides, frontal dysexecutive syndrome may potentially reveal the location of the lesion and pathophysiology of the disease process. Cranial nerve examination in the backdrop of dysexecutive syndrome should be carefully dealt with and it ranges from mere chance association to an intricate relationship with the underlying disease process and lesion location.

\section{Conclusions}

Brain tumors may cause frontal dysexecutive syndrome either from the involvement of a strategic location or from the involvement of a distant, unrelated region. They exert their impacts differently yet can give rise to similar cognitive impairments and can manifest as dementia. Known psychiatric disorders are great mimickers of this condition. Associated supranuclear eye movement abnormalities may be one of the basic valuable tools to detect the underlying disease condition. Motor and cranial nerve examination needs pragmatism as widespread variability in the nature of involvement makes an exact diagnosis more challenging.

\section{Statement of Ethics}

Our patients gave their written informed consent for publication of the article (including imaging results).

\section{Conflict of Interest Statement}

The authors have no conflicts of interest to declare.

\section{Funding Sources}

No funding was received for this work.

\section{Author Contributions}

R.G. and S.D. diagnosed the cases and were involved in the care of the patients. R.G., S.D., Sr.C., Sm.C., S.S., M.J.D., and B.K.R. were involved in writing the draft which was further modified after critical input from B.K.R., P.J.M., and J.B.-L. All authors agreed upon the final version of the article.

\section{Karger's}




\section{References}

1 Arvanitakis Z, Shah RC, Bennett DA. Diagnosis and management of dementia: review. JAMA. 2019;322(16): 1589-99.

2 Ali FS, Hussain MR, Gutiérrez C, Demireva P, Ballester LY, Zhu JJ, et al. Cognitive disability in adult patients with brain tumors. Cancer Treat Rev. 2018;65:33-40.

3 Yu Z, Tao L, Qian Z, Wu J, Liu H, Yu Y, et al. Altered brain anatomical networks and disturbed connection density in brain tumor patients revealed by diffusion tensor tractography. Int J Comput Assist Radiol Surg. 2016; 11(11):2007-19.

4 D'Onofrio G, Panza F, Sancarlo D, Addante F, Solfrizzi V, Cantarini C, et al. Executive dysfunction detected with the frontal assessment battery in Alzheimer's disease versus vascular dementia. J Alzheimers Dis. 2018;62(2): 699-711.

5 Holl AK, Wilkinson L, Tabrizi SJ, Painold A, Jahanshahi M. Selective executive dysfunction but intact risky decision-making in early huntington's disease. Mov Disord. 2013;28(8):1104-9.

6 Nowak MM, Fersten E, Głowacki M. Executive functioning pattern as a prognostic indicator for shunt implantation surgery in patients with normal pressure hydrocephalus - a preliminary report. Neurol Neurochir Pol. 2016;50(2):98-100.

7 Ou R, Liu H, Hou Y, Wei Q, Cao B, Zhao B, et al. Executive dysfunction, behavioral changes and quality of life in Chinese patients with progressive supranuclear palsy. J Neurol Sci. 2017;380:182-6.

8 Walker KA, Brown GG. HIV-associated executive dysfunction in the era of modern antiretroviral therapy: a systematic review and meta-analysis. J Clin Exp Neuropsychol. 2018;40(4):357-76.

9 Aarsen FK, Arts WF, Van Veelen-Vincent ML, Lequin MH, Catsman-Berrevoets CE. Long-term outcome in children with low grade tectal tumours and obstructive hydrocephalus. Eur J Paediatr Neurol. 2014;18(4): 469-74.

10 Schmahmann JD. The cerebellum and cognition. Neurosci Lett. 2019;688:62-75.

11 McColgan P, Tabrizi SJ. Huntington's disease. Huntington's disease: a clinical review. Eur J Neurol. 2018;25(1): 24-34.

12 Griessenauer CJ, Rizk E, Miller JH, Hendrix P, Tubbs RS, Dias MS, et al. Pediatric tectal plate gliomas: clinical and radiological progression, MR imaging characteristics, and management of hydrocephalus. J Neurosurg Pediatr. 2014;13(1):13-20.

13 Yasar S, Jusue-Torres I, Lu J, Robison J, Patel MA, Crain B, et al. Alzheimer's disease pathology and shunt surgery outcome in normal pressure hydrocephalus. PLoS One. 2017;12(8):e0182288.

14 Thai ML, Andreassen AK, Bliksted V. A meta-analysis of executive dysfunction in patients with schizophrenia: different degree of impairment in the ecological subdomains of the behavioural assessment of the dysexecutive syndrome. Psychiatry Res. 2019;272:230-6.

15 Dubey S, Ghosh R, Dubey MJ, Sengupta S, Benito-León J, Ray BK. Bilateral thalamic changes in anti-NMDAR encephalitis presenting with hemichorea and dystonia and acute transient psychotic disorder. J Neuroimmunol. 2020;347:577329.

16 Knight MJ, Baune BT. Cognitive dysfunction in major depressive disorder. Curr Opin Psychiatry. 2018;31(1): 26-31.

17 Degner D, Haust M, Meller J, Rüther E, Reulbach U. Association between autoimmune thyroiditis and depressive disorder in psychiatric outpatients. Eur Arch Psychiatry Clin Neurosci. 2015;265(1):67-72.

18 Litwin T, Dusek P, Szafrański T, Dzieżyc K, Członkowska A, Rybakowski JK. Psychiatric manifestations in Wilson's disease: possibilities and difficulties for treatment. Ther Adv Psychopharmacol. 2018;8(7):199-211.

19 Collewijn H, Went LN, Tamminga EP, Vegter-Van der Vlis M. Oculomotor defects in patients with Huntington's disease and their offspring. J Neurol Sci. 1988;86(2-3):307-20.

20 White OB, Fielding J. Cognition and eye movements: assessment of cerebral dysfunction. J Neuroophthalmol. 2012;32(3):266-73.

21 Rodríguez-Labrada R, Velázquez-Pérez L, Aguilera-Rodríguez R, Seifried-Oberschmidt C, Peña-Acosta A, Canales-Ochoa N, et al. Executive deficit in spinocerebellar ataxia type 2 is related to expanded cag repeats: evidence from antisaccadic eye movements. Brain Cogn. 2014;91:28-34.

22 Fielding J, Kilpatrick T, Millist L, White O. Multiple sclerosis: Cognition and saccadic eye movements. J Neurol Sci. 2009;277(1-2):32-6. 